\title{
Regional Brain Glucose Metabolism and Neurocognitive Function in Adult Survivors of Childhood Cancer Treated with Cranial Radiation
}

\author{
Kevin R. Krull ${ }^{1}$, Satoshi Minoshima ${ }^{2}$, Michelle Edelmann ${ }^{1}$, Brannon Morris ${ }^{3}$, Noah D. Sabin ${ }^{4}$, Tara M. Brinkman ${ }^{1}$, \\ Gregory T. Armstrong ${ }^{1}$, Leslie L. Robison ${ }^{1}$, Melissa M. Hudson ${ }^{1,5}$, and Barry Shulkin ${ }^{4}$ \\ ${ }^{1}$ Department of Epidemiology and Cancer Control, St. Jude Children's Research Hospital, Memphis, Tennessee; ${ }^{2}$ Department of \\ Radiology, University of Washington, Seattle, Washington; ${ }^{3}$ Athens Neurological Associates, Athens, Georgia; ${ }^{4}$ Department of \\ Radiological Sciences, St. Jude Children's Research Hospital, Memphis, Tennessee; and Department of Oncology, St. Jude \\ Children's Research Hospital, Memphis, Tennessee
}

\begin{abstract}
The objective of this study was to examine associations between regional brain metabolism, as measured by ${ }^{18} \mathrm{~F}-\mathrm{FDG}$ PET, and neurocognitive outcomes in adult survivors of childhood acute lymphoblastic leukemia (ALL) treated with cranial radiation. Method: Thirty-eight adult survivors of ALL were randomly selected from a large cohort treated with cranial radiation therapy (19 with 18 Gy and 19 with 24 Gy of exposure). At a mean age of 26.4 (range, 22.3-37.4) years, and 23.5 (range, 20.4-32.8) years since diagnosis, patients underwent comprehensive neurocognitive evaluations and brain ${ }^{18} \mathrm{~F}-\mathrm{FDG}$ PET imaging during a resting condition. ${ }^{18} \mathrm{~F}-\mathrm{FDG}$ PET images were analyzed stereotactically, and pixel values were normalized to global activity. Predefined region-of-interest and voxel-based correlation analyses were performed. Results: Compared with national norms, survivors demonstrated lower vocabulary $(P<0.001)$, reading $(P<0.001)$, mathematics $(P<0.001)$, working memory $(P<0.001)$, oral naming speed $(P<0.001)$, and cognitive flexibility $(P<0.001)$. Metabolic activity was higher in basal gangliar structures for those treated with 24 Gy of cranial radiation therapy $(P=$ 0.04). Metabolic activity was positively correlated with oral naming speed in both lateral frontal lobes $(\rho=0.48$ and 0.47 for right and left frontal regions, respectively, $P<0.01$ ) and negatively correlated with cognitive flexibility in the sections of the basal ganglia $(P<0.01$ for both caudate and putamen). Conclusion: Neurocognitive impairment in long-term survivors of ALL treated with cranial radiation appears to be associated with increased metabolic activity in frontal cerebral cortical and subcortical regions in the basal ganglia, suggesting decreased efficiency of the frontostriatal brain circuit.
\end{abstract}

Key Words: ${ }^{18}$ F-FDG PET; childhood cancer survivor; neurocognitive function

J Nucl Med 2014; 55:1805-1810

DOI: 10.2967/jnumed.114.142950

\footnotetext{
A

dvances in treatment of childhood cancer have resulted in survival rates exceeding $80 \%$, with current estimates suggesting 1 in 640 young adults in the general U.S. population is a survivor of

Received May 7, 2014; revision accepted Aug. 25, 2014.

For correspondence or reprints contact: Kevin R. Krull, 262 Danny Thomas Place, MS 735, Memphis, TN 38105-3678.

E-mail: kevin.krull@stjude.org

Published online Oct. 13, 2014.

COPYRIGHT (c) 2014 by the Society of Nuclear Medicine and Molecular Imaging, Inc.
}

childhood cancer (1). A significant percentage of these survivors experience treatment-related complications as they age into adulthood (2). Neurocognitive impairment, particularly in executive function, is relatively common in adult survivors of childhood acute lymphoblastic leukemia (ALL) treated with cranial radiation therapy (CRT) (3). Many of these survivors demonstrate long-term decline in neurocognitive function (4). Furthermore, we have recently demonstrated patterns of brain morphology and neurocognitive function in adult survivors of childhood ALL treated with CRT that are consistent with reports of aging adults at risk for dementia (5).

Although dose of CRT and region of exposed brain are generally associated with risk of impairment, the pathophysiology of brain response to treatment is not well understood. Prior studies have demonstrated reduced white matter volume and integrity in survivors treated with CRT (6-8). More recently, reduced volume of cortical gray matter has been reported in long-term survivors (9), though the functional significance of these differences is uncertain. To date, there have been few studies that have directly examined cortical and subcortical gray matter function.

PET is an established technique for imaging functional processes in the body. When combined with ${ }^{18}$ F-FDG (i.e., ${ }^{18} \mathrm{~F}-\mathrm{FDG}$ PET), metabolism can be examined within discrete body regions, including specific cortical and subcortical regions of the brain. Brain ${ }^{18}$ F-FDG PET has frequently been used to examine aging adults who are either at risk for or currently experiencing dementia. Glucose metabolism in frontal, parietal, and temporal lobes has been found to distinguish healthy aging adults from those diagnosed with Alzheimer disease, with $89 \%$ sensitivity and $86 \%$ specificity (10). ${ }^{18}$ F-FDG PET has also demonstrated differences between Alzheimer and frontotemporal dementia (11), the latter of which is characterized by patterns of executive dysfunction (12) similar to those observed in adult survivors of childhood ALL (3). Frontotemporal dementia appears related to dysfunction in the frontostriatal circuit (12), a network involving reciprocal connections between dorsolateral frontal lobe regions and the basal ganglia.

${ }^{18}$ F-FDG PET has been used to examine brain function in survivors of childhood cancer, with mixed results. Kahkonen et al. reported no difference in global brain activity in 20 survivors of ALL treated with CRT, compared with 20 treated with chemotherapy only (13). Similar results have been reported when examining global brain activity in survivors of central nervous system tumors treated with CRT, though when regional functioning was examined 
lower activity was identified in frontal, parietal, and temporal lobes (14). Cortical brain activity has been reported to negatively correlate with activity in subcortical regions after CRT (15). These brain ${ }^{18}$ F-FDG PET studies in childhood cancer have primarily focused on survivors who are relatively early in the phase of survivorship, with no studies of aging adult survivors of childhood cancer. In addition, the clinical significance of the differences in metabolic activity is unclear.

In the current study, we report brain ${ }^{18}$ F-FDG PET and neurocognitive function in aging adult survivors of childhood ALL who were treated with CRT at least $10 \mathrm{y}$ before current assessment. Given that these survivors are known to have deficits in executive functions (3), we expected brain metabolism in frontal regions to be associated with neurocognitive test results.

\section{MATERIALS AND METHODS}

\section{Study Population}

Participants were randomly selected from a large cohort of more than 1,400 survivors of ALL registered in the St. Jude Lifetime Cohort Study (16). Eligibility criteria for our sample included age at diagnosis of $21 \mathrm{y}$ or younger, current age $18 \mathrm{y}$ or older, and $10 \mathrm{y}$ or more from diagnosis and treatment with CRT. Survivors treated for ALL received either 18 Gy $(n=19)$ or 24 Gy $(n=19)$ of CRT. Patients were excluded if they had a history of a developmental disorder or neurologic event unrelated to their cancer history or if they relapsed or were diagnosed with a second cancer that required additional CRT or neurotoxic chemotherapy (i.e., intrathecal or high dose intravenous methotrexate). Sixty-five potentially eligible survivors were identified from medical records and randomly selected using statistical software with a random number generator. Survivors were contacted for recruitment and scheduled for campus visits. During the campus visit, 5 survivors were identified as having experienced a secondary brain tumor and 2 had a subsequent neurologic injury unrelated to their ALL diagnosis, leaving 58 truly eligible survivors. Of these, 13 refused participation, 7 withdrew before data collection, and $38(65.5 \%)$ participated in the study.

All survivors provided written informed consent, and the protocol was approved by the St. Jude Children's Research Hospital Institutional Review Board.

\section{Procedure}

Medical record abstraction was performed to capture exposure data including chemotherapy (cumulative doses), surgical procedures, and radiation (fields, doses, and beam energy). All participants were mailed comprehensive questionnaires covering health history and status, social and demographic factors, health behaviors, and psychosocial history. Each participant underwent risk-based medical assessment consistent with the Children's Oncology Group Long-Term Follow-Up Guidelines for Survivors of Childhood, Adolescent and Young Adult Cancer (17). Assessment was based on primary diagnosis, age at diagnosis, and therapeutic interventions. Survivors treated with cranial radiation or antimetabolite chemotherapy underwent neurocognitive assessment.

Neurocognitive testing was conducted during a single 2-h visit within $3 \mathrm{~d}$ of medical examination and ${ }^{18} \mathrm{~F}-\mathrm{FDG}$ PET brain imaging.

TABLE 1

Demographic and Treatment Characteristics and Performance on Neurocognitive Tests

\begin{tabular}{|c|c|c|c|c|c|c|c|c|c|}
\hline \multirow[b]{2}{*}{ Survivor characteristic } & \multicolumn{4}{|c|}{ 18-Gy ALL } & \multicolumn{4}{|c|}{ 24-Gy ALL } & \multirow[b]{2}{*}{$P^{\dagger}$} \\
\hline & Mean & SD & $\begin{array}{c}95 \% \text { confidence } \\
\text { interval }\end{array}$ & $P^{\star}$ & Mean & SD & $\begin{array}{l}95 \% \text { confidence } \\
\text { interval }\end{array}$ & $P^{\star}$ & \\
\hline Current age & 25.4 & 2.57 & $24.2-26.6$ & & 27.5 & 3.52 & $25.8-29.2$ & & 0.05 \\
\hline Age at diagnosis & 2.8 & 1.59 & $2.1-3.6$ & & 2.9 & 1.63 & $2.2-3.7$ & & 0.81 \\
\hline Time since diagnosis & 22.6 & 1.92 & 21.6-23.5 & & 24.5 & 3.03 & 23.0-25.9 & & 0.03 \\
\hline \multicolumn{10}{|l|}{ Neurocognitive functions } \\
\hline Global intelligence & -0.38 & 0.81 & -0.77 to 0.01 & 0.06 & -0.36 & 0.79 & -0.74 to 0.02 & 0.06 & 0.96 \\
\hline Vocabulary & -0.83 & 1.09 & -1.35 to -0.30 & 0.004 & -0.74 & 1.06 & -1.25 to -0.23 & 0.007 & 0.81 \\
\hline Word reading & -0.53 & 0.55 & -0.80 to -0.27 & $<0.001$ & -0.49 & 0.55 & -0.76 to -0.22 & 0.001 & 0.80 \\
\hline Calculations & -0.74 & 0.89 & -1.17 to -0.31 & 0.002 & -0.67 & 0.65 & -0.99 to -0.36 & $<0.001$ & 0.79 \\
\hline Visuomotor speed & -0.56 & 0.69 & -0.89 to -0.23 & 0.02 & -0.40 & 0.58 & -0.69 to -0.12 & 0.007 & 0.45 \\
\hline Oral naming speed & -0.91 & 0.77 & -1.28 to -0.54 & $<0.001$ & -0.98 & 0.91 & -1.42 to -0.54 & $<0.001$ & 0.79 \\
\hline Focused attention & -0.27 & 0.89 & -0.70 to 0.16 & 0.20 & -0.06 & 1.45 & -0.75 to 0.64 & 0.87 & 0.59 \\
\hline Sustained attention & -0.52 & 1.09 & -1.04 to 0.01 & 0.06 & -0.18 & 0.79 & -0.56 to 0.20 & 0.34 & 0.28 \\
\hline Attention span & -0.87 & 0.91 & -1.31 to -0.43 & 0.001 & -0.53 & 1.04 & -1.04 to -0.03 & 0.04 & 0.30 \\
\hline Verbal memory & -0.77 & 1.03 & -1.27 to -0.28 & 0.004 & -1.19 & 0.92 & -1.64 to -0.75 & $<0.001$ & 0.19 \\
\hline Working memory & -0.39 & 0.66 & -0.70 to -0.07 & 0.02 & -0.39 & 0.76 & -0.75 to -0.03 & 0.04 & 0.99 \\
\hline Cognitive flexibility & -0.88 & 1.49 & -1.60 to -0.16 & 0.02 & -1.03 & 1.33 & -1.67 to -0.39 & 0.003 & 0.75 \\
\hline Cognitive fluency & -0.12 & 0.84 & -0.53 to 0.28 & 0.53 & -0.51 & 0.88 & -0.93 to -0.07 & 0.02 & 0.18 \\
\hline
\end{tabular}

${ }^{*} P$ values for 1 -sample $t$ tests comparing mean to value of 0.0 .

${ }^{\dagger} P$ values for 2 group $t$ tests.

Values for neurocognitive functions are presented in age-adjusted $z$ scores (mean $=0, S D=1.0$ ), based on national normative data; $95 \%$ confidence intervals for these scores that do not contain value of 0.0 indicate that mean for respective group falls significantly below expected value. 
Assessed domains included intelligence (18), academic skills (19), attention (20-22), memory (23), processing speed (20-22), and executive function $(20,21)$. Survivors completed a self-rating questionnaire to evaluate perceived neurobehavioral function, which has been validated in numerous studies of medical illness and neurologic injury and includes separate indices of behavioral and cognitive problems (24). Order of testing was standardized, and survivors' schedules were adjusted to limit impact from fatigue and extraneous factors.

For PET/CT examinations, $55.5 \mathrm{MBq} / \mathrm{kg}(0.15 \mathrm{mCi} / \mathrm{kg})$ of ${ }^{18} \mathrm{~F}-\mathrm{FDG}$ (maximum, $444 \mathrm{MBq}(12 \mathrm{mCi})]$ were injected intravenously in patients after an overnight fast or after a minimum 4-h fast for studies done in the afternoon. Patients stayed in a quiet, dark room after the injection and were encouraged to remain recumbent and relaxed. Transmission CT images for attenuation correction and lesion localization as well as PET emission images were acquired approximately $1 \mathrm{~h}$ later using a Discovery LS PET/CT system (GE Healthcare); in later studies, a Discovery LS 690 (GE Healthcare) was used. CT parameters were helical acquisition; tube rotation, $0.5 \mathrm{~s}$; coverage, $39.27 \mathrm{~mm} /$ rotation; pitch, 0.984:1; slice thickness, $3.75 \mathrm{~mm}$; interval, $3.27 \mathrm{~mm} ; 120 \mathrm{kV} ; 100 \mathrm{~mA}$; and noise index, 25.00 .

\section{Statistical Analyses}

Descriptive statistics were calculated for demographic and treatment characteristics and neurocognitive outcomes. The impact of CRT on neurocognitive outcomes was assessed by classifying survivors into 2 groups based on original treatment: 18 Gy or 24 Gy CRT for ALL.

Neurocognitive scores were transformed into age-adjusted $z$ scores (mean, $0 ; \mathrm{SD}, 1.0$ ) using national norms. One-sample $t$ tests were used to determine whether the mean for each group differed from the expected normal population mean of $z=0.0$. Two-sample $t$ tests were used to test for differences between groups.

${ }^{18}$ F-FDG PET images were analyzed stereotactically, and pixel values were normalized to global activity. Activity levels in predefined regions of interest (ROIs) were examined between groups using 2 -sample $t$ tests. ROIs were examined in relation to predetermined neurocognitive measures. Voxel-based correlation analyses were also conducted using statistical parametric mapping, and associations with neurocognitive function were examined. Only neurocognitive measures that demonstrated either group differences or differences from the expected normal distribution in each group were used in the ROIand voxel-based correlation analyses.

\section{RESULTS}

Ten female and 9 male survivors were included in each of the treatment exposure groups. Survivors treated with 18-Gy CRT (25.4 y) were slightly younger than survivors treated with 24-Gy CRT (27.5 y), though no difference was observed in age at diagnosis (Table 1). This difference in current age and an observed difference in time since diagnosis (22.6 and 24.5 y for 18 - and $24-\mathrm{Gy}$ CRT groups, respectively; $P=0.03$ ) reflects the historic change from 24- to 18-Gy CRT for prophylactic treatment of the central nervous system in standard-risk ALL. Both groups demonstrated significant impairment in multiple neurocognitive functions when compared with normative data, though no differences were apparent between groups for any of the neurocognitive outcomes (Table 1). In particular, lower age-adjusted scores were observed on measures of vocabulary, reading, mathematics, visuomotor speed, oral naming speed, attention span, verbal memory, working memory, and cognitive flexibility.

Table 2 presents ${ }^{18} \mathrm{~F}$-FDG PET activation values by predetermined brain ROIs. With regions normalized to global brain activity, both groups demonstrated relatively higher activity in the cerebral cortex, basal ganglia, and thalamic regions. Relatively lower activity was observed in the cerebellum, cingulate gyrus, amygdala, and parahippocampal regions. Only activity in the putamen differed significantly between groups, though the effect size correlation coefficients $(\mathrm{d} / \sqrt{ }[\mathrm{d} 2+4])$ were modest for both the right $(0.32)$ and the left $(0.34)$ putamen.

Given the lack of group differences in neurocognitive functions (Table 1) and similar patterns in ${ }^{18} \mathrm{~F}$-FDG PET activation levels (Table 2), groups were combined for subsequent analysis of associations between these outcomes. Correspondence was examined between those neurocognitive functions with significant rates of impairment and brain ROIs (Table 3). Slower oral naming speed was associated with lower metabolism in bilateral frontal lobes, whereas lower cognitive flexibility was associated with lower metabolism in bilateral parietal lobes. Increased metabolic activity in the striate (caudate and putamen) was associated with problems in multiple cognitive functions, including cognitive flexibility, working memory, attention span, reading, and mathematic calculations.

TABLE 2

${ }^{18}$ F-FDG PET Values for Predetermined ROls in Brain

\begin{tabular}{|c|c|c|c|c|c|}
\hline \multirow[b]{2}{*}{$\mathrm{ROI}$} & \multicolumn{2}{|c|}{ 18-Gy ALL } & \multicolumn{2}{|c|}{ 24-Gy ALL } & \multirow[b]{2}{*}{$P$} \\
\hline & Mean & SD & Mean & SD & \\
\hline Lateral frontal lobe (R) & 1.15 & 0.03 & 1.14 & 0.03 & 0.41 \\
\hline Lateral frontal lobe $(L)$ & 1.13 & 0.03 & 1.11 & 0.03 & 0.20 \\
\hline Medial frontal lobe $(\mathrm{R})$ & 1.04 & 0.02 & 1.02 & 0.03 & 0.08 \\
\hline Medial frontal lobe (L) & 1.03 & 0.03 & 1.02 & 0.03 & 0.12 \\
\hline Lateral parietal lobe (R) & 1.09 & 0.03 & 1.08 & 0.03 & 0.65 \\
\hline Lateral parietal lobe (L) & 1.07 & 0.03 & 1.06 & 0.04 & 0.25 \\
\hline Medial parietal lobe $(\mathrm{R})$ & 1.07 & 0.03 & 1.07 & 0.04 & 0.69 \\
\hline Medial parietal lobe (L) & 1.07 & 0.04 & 1.07 & 0.06 & 0.66 \\
\hline Occipital lobe (R) & 1.14 & 0.04 & 1.16 & 0.05 & 0.20 \\
\hline Occipital lobe (L) & 1.11 & 0.04 & 1.12 & 0.05 & 0.63 \\
\hline Temporal lobe (R) & 1.04 & 0.03 & 1.04 & 0.03 & 0.94 \\
\hline Temporal lobe (L) & 1.01 & 0.03 & 1.02 & 0.03 & 0.71 \\
\hline Parahippocampus (R) & 0.72 & 0.04 & 0.74 & 0.03 & 0.11 \\
\hline Parahippocampus (L) & 0.73 & 0.03 & 0.73 & 0.03 & 0.36 \\
\hline Anterior cingulate gyrus (R) & 0.92 & 0.05 & 0.91 & 0.04 & 0.63 \\
\hline Anterior cingulate gyrus $(\mathrm{L})$ & 0.92 & 0.05 & 0.91 & 0.04 & 0.28 \\
\hline Posterior cingulate gyrus (R) & 1.08 & 0.05 & 1.08 & 0.03 & 0.93 \\
\hline Posterior cingulate gyrus (L) & 1.06 & 0.05 & 1.06 & 0.04 & 0.92 \\
\hline Cerebellum (R) & 0.89 & 0.05 & 0.89 & 0.06 & 0.85 \\
\hline Cerebellum (L) & 0.88 & 0.05 & 0.88 & 0.06 & 0.94 \\
\hline Caudate (R) & 1.22 & 0.10 & 1.23 & 0.07 & 0.84 \\
\hline Caudate (L) & 1.21 & 0.07 & 1.23 & 0.07 & 0.26 \\
\hline Putamen (R) & 1.08 & 0.06 & 1.12 & 0.06 & 0.05 \\
\hline Putamen (L) & 1.09 & 0.07 & 1.14 & 0.07 & 0.03 \\
\hline Amygdala (R) & 0.63 & 0.04 & 0.65 & 0.05 & 0.20 \\
\hline Amygdala (L) & 0.64 & 0.05 & 0.66 & 0.04 & 0.45 \\
\hline Thalamus (R) & 1.03 & 0.06 & 1.05 & 0.04 & 0.27 \\
\hline Thalamus (L) & 1.04 & 0.06 & 1.06 & 0.05 & 0.17 \\
\hline
\end{tabular}

Values normalized to global PET activity. 
TABLE 3

Correlation Between Neurocognitive Function and ${ }^{18}$ F-FDG PET Brain ROI

\begin{tabular}{|c|c|c|c|c|c|c|c|c|c|}
\hline \multirow[b]{2}{*}{ ROI } & \multicolumn{9}{|c|}{ Neurocognitive function } \\
\hline & Vocabulary & Reading & Calculation & Visuomotor & $\begin{array}{c}\text { Oral } \\
\text { naming }\end{array}$ & $\begin{array}{l}\text { Attention } \\
\text { span }\end{array}$ & $\begin{array}{l}\text { Verbal } \\
\text { memory }\end{array}$ & $\begin{array}{l}\text { Working } \\
\text { memory }\end{array}$ & $\begin{array}{l}\text { Cognitive } \\
\text { flexibility }\end{array}$ \\
\hline Lateral frontal lobe $(\mathrm{R})$ & 0.06 & -0.20 & 0.05 & -0.11 & $0.48^{\star}$ & -0.07 & 0.10 & -0.05 & 0.13 \\
\hline Lateral frontal lobe (L) & 0.20 & -0.09 & 0.01 & -0.11 & $0.47^{\dagger}$ & -0.13 & 0.20 & 0.02 & -0.08 \\
\hline Medial frontal lobe (R) & -0.01 & -0.28 & 0.21 & -0.04 & -0.17 & -0.10 & 0.12 & -0.17 & 0.14 \\
\hline Medial frontal lobe (L) & 0.19 & -0.05 & 0.27 & 0.03 & -0.07 & -0.12 & 0.23 & 0.00 & 0.15 \\
\hline Lateral parietal lobe $(\mathrm{R})$ & -0.19 & -0.03 & -0.29 & 0.09 & 0.09 & -0.07 & -0.06 & 0.14 & $0.40^{\ddagger}$ \\
\hline Lateral parietal lobe (L) & -0.01 & 0.10 & -0.09 & 0.16 & 0.16 & -0.10 & 0.29 & -0.01 & $0.36^{\ddagger}$ \\
\hline Medial parietal lobe (R) & 0.04 & 0.16 & -0.21 & -0.14 & 0.07 & -0.10 & 0.27 & 0.16 & 0.19 \\
\hline Medial parietal lobe (L) & 0.05 & 0.23 & -0.10 & -0.10 & 0.17 & 0.24 & 0.26 & 0.18 & 0.22 \\
\hline Temporal lobe (R) & 0.33 & 0.27 & 0.18 & 0.20 & -0.04 & -0.03 & 0.15 & $0.37^{\ddagger}$ & -0.06 \\
\hline Temporal lobe (L) & 0.36 & 0.32 & 0.19 & 0.26 & 0.08 & -0.06 & 0.29 & 0.20 & -0.07 \\
\hline Cerebellum (R) & -0.09 & -0.05 & -0.18 & -0.18 & -0.10 & 0.22 & 0.11 & 0.17 & -0.17 \\
\hline Cerebellum (L) & -0.14 & -0.06 & -0.21 & -0.16 & -0.05 & 0.19 & 0.02 & 0.11 & -0.16 \\
\hline Caudate (R) & -0.24 & -0.30 & $-0.43^{\dagger}$ & -0.20 & -0.16 & -0.32 & -0.13 & $-0.57^{\star}$ & $-0.56^{\star}$ \\
\hline Caudate (L) & -0.11 & -0.32 & -0.32 & -0.12 & -0.31 & $-0.39 \ddagger$ & -0.08 & $-0.51^{*}$ & $-0.47^{\dagger}$ \\
\hline Putamen (R) & -0.35 & $-0.39 \ddagger$ & $-0.45^{\dagger}$ & -0.14 & -0.16 & $-0.35^{\ddagger}$ & -0.33 & $-0.53^{*}$ & $-0.56^{\star}$ \\
\hline Putamen (L) & -0.31 & $-0.40^{\ddagger}$ & $-0.39 \ddagger$ & -0.10 & -0.14 & $-0.38^{\ddagger}$ & $-0.36^{\ddagger}$ & $-0.50^{\star}$ & $-0.56^{\star}$ \\
\hline Amygdala (R) & 0.08 & 0.17 & 0.11 & -0.09 & 0.23 & 0.02 & -0.15 & 0.05 & 0.03 \\
\hline Amygdala (L) & 0.09 & 0.07 & 0.04 & -0.33 & 0.03 & 0.05 & 0.03 & 0.22 & -0.23 \\
\hline Parahippocampus (R) & -0.03 & -0.03 & -0.05 & 0.02 & 0.15 & -0.04 & -0.14 & 0.12 & 0.09 \\
\hline Parahippocampus (L) & -0.01 & 0.19 & -0.10 & -0.21 & 0.28 & 0.05 & -0.22 & 0.19 & -0.06 \\
\hline
\end{tabular}

Correlation coefficients presented for entire sample by right and left brain $\mathrm{ROI}$, with significance identified as follows: ${ }^{*} P<0.005$; ${ }^{\dagger} P<0.01$; $\ddagger P<0.05$.

Figures 1 and 2 demonstrate voxel-based correlation between brain glucose metabolism and oral naming and cognitive flexibility, respectively.

\section{DISCUSSION}

The pattern of neurocognitive and brain imaging results displayed in this study suggest functional impairment predominantly in frontotemporal brain regions. Compared with age-adjusted norms, survivors demonstrated lower performance on neurocognitive processes associated with frontal lobe brain regions, including measures of cognitive flexibility, fluency, working memory, attention span, and oral naming speed. Lower performance was also observed on measures of verbal memory, vocabulary, and reading, skills associated with temporal and frontal brain regions. Many of these neurocognitive functions were correlated with resting-state glucose metabolism in lateral frontal brain regions, as well as subcortical centers in the basal ganglia (i.e., caudate and putamen). The basal ganglia have numerous projections to frontal (25) and temporal cortices (25), predominantly through the frontostriatal circuit (12). Taken as a whole, this pattern suggests disrupted development or activation in this circuit.

Differential associations between cortical-subcortical metabolism were observed with neurocognitive function. Lower glucose metabolism in the cerebral cortex was associated with lower neurocognitive function, whereas higher glucose metabolism in the striatum was associated with lower neurocognitive function. This pattern is likely a result of the reciprocal cortical-subcortical feedback circuits that exist in the human brain (26). Cortical regions in the frontal lobes not only receive input from the basal ganglia, but also serve to regulate these subcortical structures to control complex information processing, decision making, and planned actions (27). This feedback loop likely accounts for the negative correlation between cortical and subcortical regions after CRT that was previously reported in 12 survivors of childhood ALL (15). Increased activation in subcortical regions, due presumptively to reduced inhibition from cortical regions, may affect the efficiency of neurocognitive processes, particularly executive functions.

The pattern of brain function seen in adult survivors of childhood ALL treated with CRT appears to involve the frontal and temporal lobes, as well as the deep gray nuclei, and suggests involvement of the frontostriatal circuit. However, a diagnosis of frontotemporal dementia is not warranted. In addition to impaired executive function and memory, frontotemporal dementia is associated with a pattern of changes in behavior, including emotional lability and disinhibition (12). Adult survivors of childhood ALL do have high rates of emotional dysregulation (3), though there is no evidence of generalized impulsivity or disinhibition. Behavioral disinhibition is often associated with orbitomedial regions in the frontal lobes, whereas cognitive flexibility and working memory are associated with the dorsolateral frontal lobes (12). Thus, a more specific frontal brain circuit appears compromised in the adult survivors of ALL. 

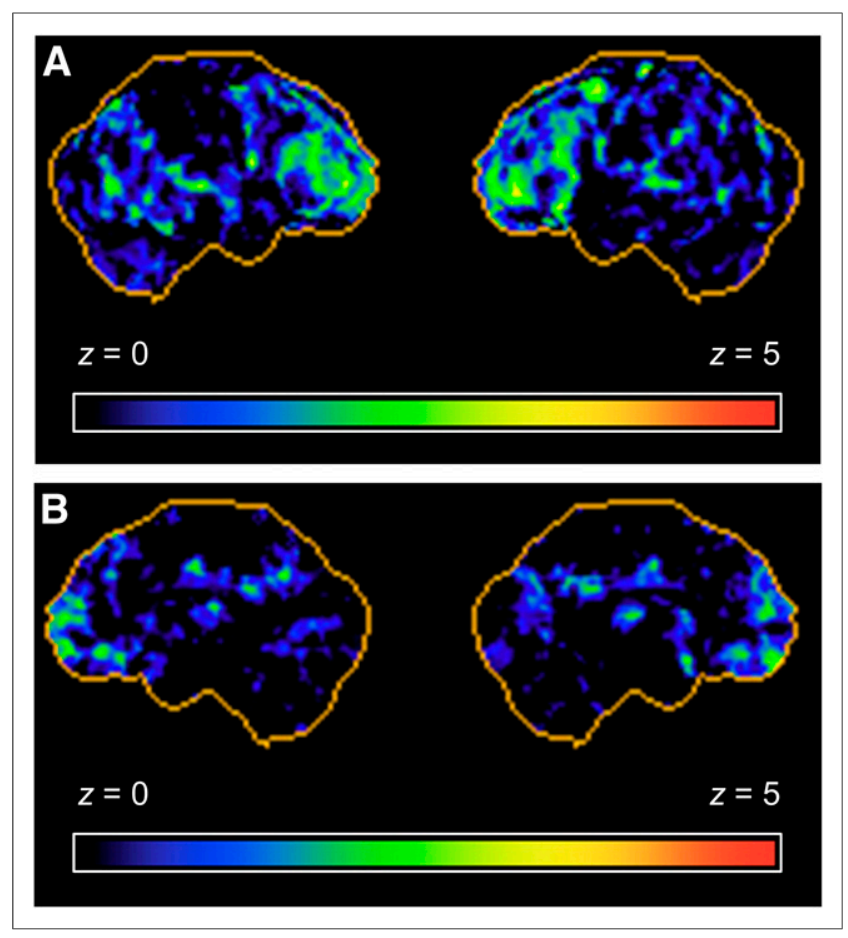

FIGURE 1. Lateral (A) and medial (B) views of voxel-based correlation analysis between resting-state brain ${ }^{18} \mathrm{~F}-\mathrm{FDG}$ PET activity and ageadjusted performance on oral naming task. Metabolic activity in lateral frontal lobes was correlated with performance on naming test. $z$ scores are represented on color-coded scale ranging from 0 (black) to 5 (red).

Previous brain imaging research has implicated white matter as a primary focus of disruption after cancer therapy for childhood ALL (28). The current results point to a prominent impact on cortical and subcortical gray matter as well. This involvement of the cerebral cortex is supported by recent quantitative brain imaging studies. Zeller et al. demonstrated that adult survivors of ALL had a smaller volume of cortical gray matter and basal ganglia than healthy controls (9). We recently extended these results by demonstrating associations between impaired memory function and frontal and temporal cortical thickness as well as smaller hippocampal volume in adult survivors (5). Further research is needed to examine interconnections among networks of cortical and subcortical brain regions as well as how these network influence functional outcomes.

This study is not without limitations. The sample size was relatively small, compared with recent studies in healthy aging and Alzheimer dementia. Still, our study is the largest study to date examining ${ }^{18} \mathrm{~F}$-FDG PET in adult survivors of childhood ALL. A healthy control group was not available for comparison of ${ }^{18}$ F-FDG PET uptake or differential associations between metabolism and neurocognitive function, though population norms were available for classification of neurocognitive performance. The study involved cross-sectional assessment with no longitudinal follow-up. As such, it is not clear whether patterns of impairment reflect recent changes, long-standing deficits, or a combination of both. Longitudinal follow-up is needed to examine the evolution of the functional problems. Finally, ${ }^{18}$ F-FDG PET studies were completed during a resting sate and not during activation of specific brain functions, limiting us in directly tying brain regions to neurocognitive impairment. However, our resting-state study does permit examination of associations between general-state levels of activation and numerous different neurocognitive functions.
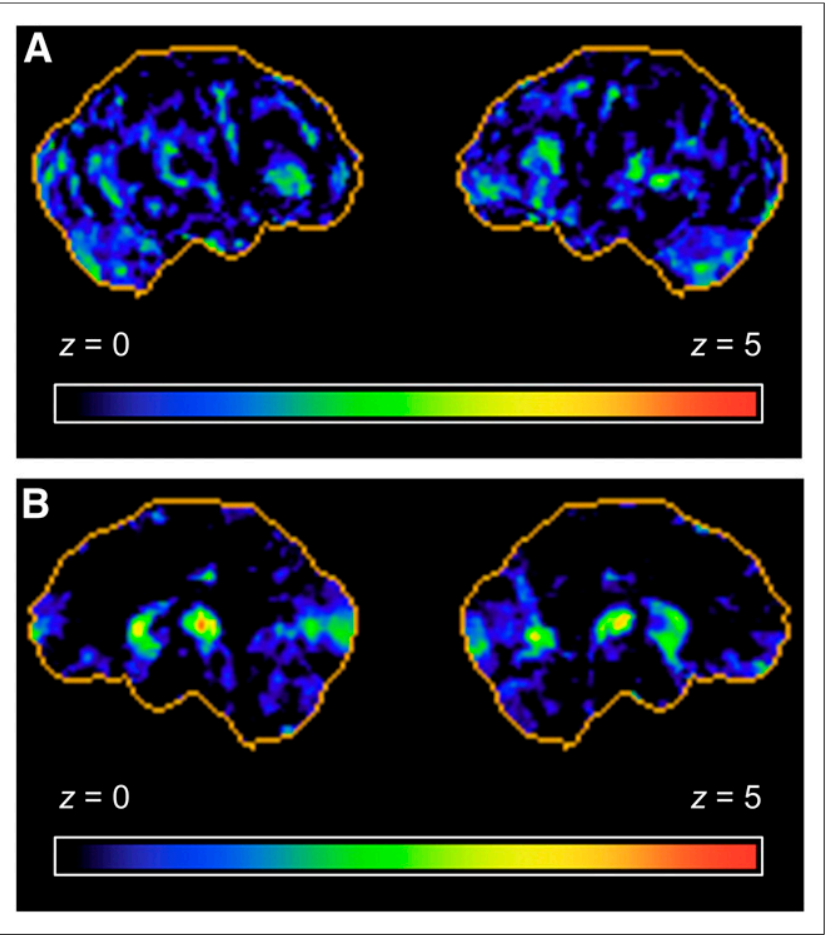

FIGURE 2. Lateral (A) and medial (B) views of voxel-based correlation analysis between resting-state brain ${ }^{18} \mathrm{~F}-\mathrm{FDG}$ PET activity and ageadjusted performance on cognitive flexibility task. Higher metabolic activity in striatum (caudate and putamen) of basal ganglia was correlated with lower cognitive flexibility. $z$ scores are represented on color-coded scale ranging from 0 (black) to 5 (red).

\section{CONCLUSION}

The current results suggest survivors treated with cranial radiation for childhood ALL develop neurocognitive patterns consistent with altered functioning in the frontostriatal brain circuit. Not only were cortical brain regions associated with neurocognitive function, so too were subcortical regions including the putamen and caudate nucleus of the basal ganglia. This finding is significant because it implicated not only cortical brain regions, but also subcortical structures in the basal ganglia as well, in this case the putamen and caudate nucleus. Identification of neural systems and subcortical nuclei can contribute to our understanding of pathophysiology in cancer survivors and may eventually lead to the identification of neurotransmitters to target for pharmacotherapeutic interventions.

\section{DISCLOSURE}

The costs of publication of this article were defrayed in part by the payment of page charges. Therefore, and solely to indicate this fact, this article is hereby marked "advertisement" in accordance with 18 USC section 1734 . This work was supported by the Cancer Center Support (CORE) grant CA21765 from the National Cancer Institute and American Lebanese Syrian Associated Charities (ALSAC). No other potential conflict of interest relevant to this article was reported.

\section{REFERENCES}

1. Howlander N, Noone AM, Krapcho M, et al. SEER cancer statistics review, 1975-2008. Surveillance, Epidemiology, and End Results, National Cancer Institute website. http://seer.cancer.gov/csr/1975_2008/. 2011. Accessed September 4, 2014. 
2. Hudson MM, Ness KK, Gurney JG, et al. Clinical ascertainment of health outcomes among adults treated for childhood cancer. JAMA. 2013;309: 2371-2381.

3. Krull KR, Brinkman TM, Li C, et al. Neurocognitive outcomes decades after treatment for childhood acute lymphoblastic leukemia: a report from the St. Jude Lifetime Cohort Study. J Clin Oncol. 2013;31:4407-4415.

4. Krull KR, Zhang N, Santucci A, et al. Long-term decline in intelligence among adult survivors of childhood acute lymphoblastic leukemia treated with cranial radiation. Blood. 2013;122:550-553.

5. Armstrong GT, Reddick WE, Petersen RC, et al. Evaluation of memory impairment in aging adult survivors of childhood acute lymphoblastic leukemia treated with cranial radiotherapy. J Natl Cancer Inst. 2013;105:899-907.

6. Shan ZY, Liu JZ, Glass JO, Gajjar A, Li CS, Reddick WE. Quantitative morphologic evaluation of white matter in survivors of childhood medulloblastoma. Magn Reson Imaging. 2006;24:1015-1022.

7. Mabbott DJ, Noseworthy MD, Bouffet E, Rockel C, Laughlin S. Diffusion tensor imaging of white matter after cranial radiation in children for medulloblastoma: correlation with IQ. Neuro-oncol. 2006;8:244-252.

8. Dellani PR, Eder S, Gawehn J, et al. Late structural alterations of cerebral white matter in long-term survivors of childhood leukemia. J Magn Reson Imaging. 2008;27:1250-1255.

9. Zeller B, Tamnes CK, Kanellopoulos A, et al. Reduced neuroanatomic volumes in long-term survivors of childhood acute lymphoblastic leukemia. J Clin Oncol. 2013;31:2078-2085.

10. Newberg AB, Arnold SE, Wintering N, Rovner BW, Alavi A. Initial clinical comparison of ${ }^{18} \mathrm{~F}$-florbetapir and ${ }^{18} \mathrm{~F}$-FDG PET in patients with Alzheimer disease and controls. J Nucl Med. 2012;53:902-907.

11. Woost TB, Dukart J, Frisch S, et al. Neural correlates of the DemTect in Alzheimer's disease and frontotemporal lobar degeneration: a combined MRI \& ${ }^{18}$ F-FDG PET study. Neuroimage Clin. 2013;2:746-758.

12. Looi JC, Walterfang M, Velakoulis D, Macfarlane MD, Svensson LA, Wahlund LO. Frontotemporal dementia as a frontostriatal disorder: neostriatal morphology as a biomarker and structural basis for an endophenotype. Aust NZ J Psychiatry. 2012;46:422-434.

13. Kähkönen M, Metsahonkala L, Minn H, et al. Cerebral glucose metabolism in survivors of childhood acute lymphoblastic leukemia. Cancer. 2000;88:693-700.
14. Andersen PB, Krabbe K, Leffers AM, et al. Cerebral glucose metabolism in long-term survivors of childhood primary brain tumors treated with surgery and radiotherapy. J Neurooncol. 2003;62:305-313.

15. Phillips PC, Moeller JR, Sidtis JJ, et al. Abnormal cerebral glucose metabolism in long-term survivors of childhood acute lymphocytic leukemia. Ann Neurol. 1991;29:263-271.

16. Hudson MM, Ness KK, Nolan VG, et al. Prospective medical assessment of adults surviving childhood cancer: study design, cohort characteristics, and feasibility of the St. Jude Lifetime Cohort Study. Pediatr Blood Cancer. 2011;56:825-836.

17. Landier W, Bhatia S, Eshelman DA, et al. Development of risk-based guidelines for pediatric cancer survivors: the Children's Oncology Group Long-Term Follow-Up Guidelines from the Children's Oncology Group Late Effects Committee and Nursing Discipline. J Clin Oncol. 2004;22:4979-4990.

18. Wechsler D. Wechsler Abbreviated Scale of Intelligence. San Antonio, TX: Psychological Corporation; 1999.

19. Woodcock RW, McGrew KS, Mather N. Woodcock-Johnson III: Tests of Achievement. Itasca, IL: Riverside; 2001.

20. Wechsler D. Wechsler Adult Intelligence Scale: Third ed. San Antonio, TX: Psychological Corporation; 1997.

21. Strauss E, Sherman EM, Spreen O. A Compendium of Neuropsychological Tests: Administration, Norms, and Commentary. 3d ed. New York, NY: Oxford University Press; 2006:655-672.

22. Conners CK. Conners' Continuous Performance Test II. Noth Tonawanda, NY: Multi-Health Systems, Inc.; 2001.

23. Delis DC, Kramer JH, Kaplan E, Ober BA. California Verbal Learning Test: Second ed. San Antonio, TX: The Psychological Corporation; 2000.

24. Roth RM, Isquith PK, Gioia GA. Behavior Rating Inventory of Executive Function: Adult Version. Lutz, FL: Psychological Assessment Resources, Inc.; 2005.

25. Middleton FA, Strick PL. The temporal lobe is a target of output from the basal ganglia. Proc Natl Acad Sci USA. 1996;93:8683-8687.

26. Lewis MD, Todd RM. The self-regulating brain: cortical-subcortical feedback and the development of intelligent action. Cogn Dev. 2007;22:406-430.

27. Casey BJ, Durston S, Fossella JA. Evidence for a mechanistic model of cognitive control. Clin Neurosci Res. 2001;1:267-282.

28. Reddick WE, Glass JO, Langston JW, Helton KJ. Quantitative MRI assessment of leukoencephalopathy. Magn Reson Med. 2002;47:912-920. 